\title{
Neoadjuvant programmed cell death protein 1 inhibitors combined with chemotherapy in resectable non-small cell lung cancer: an open-label, multicenter, single-arm study
}

\author{
Hongtao Duan ${ }^{1 \#}$, Tianhu Wang ${ }^{2 *}$, Zhilin Luo $^{2 \#}$, Liping Tong ${ }^{1}$, Xiaoping Dong ${ }^{1}$, Yong Zhang ${ }^{1}$, \\ Muhammad Zubair Afzal ${ }^{3}$, Pierpaolo Correale ${ }^{4}$, Honggang Liu ${ }^{1}$, Tao Jiang ${ }^{1}$, Xiaolong Yan ${ }^{1}$ \\ ${ }^{1}$ Department of Thoracic Surgery, Tangdu Hospital, Air Force Military Medical University, Xi'an, China; ${ }^{2}$ Department of Thoracic Surgery, Third \\ Affiliated Hospital, Chongqing Medical University, Chongqing, China; ${ }^{3}$ Hematology-Oncology, Dartmouth-Hitchcock Medical Center, Medical \\ Center Dr., Lebanon, NH, USA; ${ }^{4}$ Medical Oncology Unit, Grand Metropolitan Hospital "Bianchi-Melacrino-Morelli”, Reggio Calabria, Italy \\ Contributions: (I) Conception and design: H Duan, X Yan; (II) Administrative support: T Wang, T Jiang; (III) Provision of study materials or patients: \\ Z Luo, H Liu; (IV) Collection and assembly of data: X Dong, Y Zhang, X Yan; (V) Data analysis and interpretation: L Tong, MZ Afzal, P Correale, \\ H Duan; (VI) Manuscript writing: All authors; (VII) Final approval of manuscript: All authors. \\ "These authors equally contributed to this work. \\ Correspondence to: Professor Xiaolong Yan; Professor Tao Jiang; Professor Honggang Liu. Department of Thoracic Surgery, Tangdu Hospital, Air \\ Force Military Medical University, 1 Xinsi Road, Xi'an, China. Email: yanxiaolong558@126.com; jiangtaochest@163.com; drliu321@163.com.
}

Background: Neoadjuvant therapy has significantly improved the 5-year overall survival (OS)of patients with resectable non-small cell lung cancer (NSCLC). The CheckMate 159 trial showed that neoadjuvant therapy with a single-drug programmed cell death protein 1 (PD-1) inhibitor (nivolumab) achieved major pathological response (MPR) and pathological complete response (pCR) in $45 \%$ and $15 \%$ of participants, respectively. We conducted an open-label single-arm study to evaluate the safety and efficacy of neoadjuvant PD-1 inhibitors in combination with chemotherapy in the treatment of resectable NSCLC.

Methods: This study was conducted in a total of 2 hospitals in the Chinese cities of Xi'an and Chongqing, and included eligible patients over 18 years of age with clinically staged IIA-IIIB NSCLC. All patients were scheduled to receive surgery within 4-6 weeks after neoadjuvant treatment (3-4 cycles) consisting of PD-1 inhibitors combined with a conventional chemotherapy regimen on day 1 of each 21-day cycle.

Results: Twenty-three patients, 22 males, and 1 female with just one of them with no smoking habits) were diagnosed with NSCL C in a stage IIA (3 cases), IIB (3 cases), IIIA (8 cases), and IIIB (9cases) and no druggable driver mutations/translocations were addressed to receive neoadjuvant treatment between June 2018 and June 2020. The treatment was well tolerated with just 3 typical immune-related adverse events (hyperthyroidism, hyperglycemia, and rash) recorded. There was a partial response (PR) and stable disease (SD) in $17(73.9 \%)$ and $6(26.1 \%)$ patients, with an overall response rate (ORR) of $73.9 \%$ according to the Response Evaluation Criteria in Solid Tumors (RECIST v.1.1). Six of these patients resulted in pCR (30\%) while ten of them showed a MPR (50\%). Twenty patients underwent surgical resection after treatment, while further 3 refused surgery. Surgical procedure included video-assisted thoracoscopic resection (10 cases), Vinci Robot surgery (4 cases), and thoracotomy in 4 cases while there were secondary compliance-related thoracotomy in two cases. The pathology analysis revealed a R0 in 19 cases (19/20, 95\%).

Conclusions: Our results suggest that the neoadjuvant approach with chemotherapy and PD-1 blocking $\mathrm{mAbs}$ is safe and active in patients with resectable NSCLC where is associated with a promising high ORR, MPR and pCR.

Keywords: Neoadjuvant; programmed cell death protein 1 inhibitor combined with chemotherapy (PD-1 inhibitor combined with chemotherapy); pathological complete response (pCR); major pathological response (MPR); safety and efficacy 
Submitted Oct 19, 2020. Accepted for publication Feb 24, 2021.

doi: $10.21037 /$ tlcr-21-130

View this article at: http://dx.doi.org/10.21037/tlcr-21-130

\section{Introduction}

Neoadjuvant therapy is a promising treatment strategy for patients with resectable non-small cell lung cancer (NSCLC). This kind of treatment for many years has included chemotherapy, tyrosine kinase inhibitors (TKIs), radiotherapy, alone or in multiple combinations (1). A meta-analysis of data from NSCLC patients treated with neoadjuvant chemotherapy showed an absolute improvement in 5-year overall survival (OS) of $5 \%$ (40-45\%) (2). The mortality risk was significantly reduced in patients who achieved a major pathological response (MPR) after neoadjuvant therapy. Similarly, the results of various clinical trials in patients with bladder, breast, and gastroesophageal cancer showed an improved OS in those who achieved a pathological complete response (pCR) after neoadjuvant therapies (3). In this context further exploration concerning innovative neoadjuvant strategies in resectable NSCLC patients is strongly required.

In more recent years, immunocheckpoint blockade with mAbs to PD-1/PD-L1 alone or in combination with platinum doublets has been shown to significant efficacy in lung cancer patients. The KEYNOTE189 trial in particular, reported the efficacy of an anti-PD-1 $\mathrm{mA}$, pembrolizumab, in combination with chemotherapy in patients with advanced Lung adenocarcinoma. In particular, this trial showed a 12 -month OS of $69.2 \%$ in the experimental group compared with a $49.4 \%$ reported in the group of patients who received the chemotherapy alone (4). Furthermore, in the KEYNOTE407 trial, the OS reached 15.9 months for patients with advanced squamous cell carcinoma in the chemo-immunotherapy arm, compared with 11.3 months recorded in the group addressed to chemotherapy alone (5). The above-mentioned trials have established the role of PD-1 blockade and chemotherapy in the treatment of NSCLC. In line with these results a recent meta-analysis showed that NSCLC patients over-expressing with the programmed death ligand 1 (PD-L1) (PD-L1 score $>50 \%$ ) receiving pembrolizumab-chemotherapy combination showed a much greater ORR, and progression-free survival (PFS) compared with the groups of patients receiving chemotherapy- or pembrolizumab-alone (6). Finally, the CheckMate159 trial reported an MPR rate of $45 \%$ in NSCLC patients receiving neoadjuvant therapy with nivolumab, which was much higher than that achieved by any previously tested chemotherapy regimens in neoadjuvant setting (7). The trial therefore suggested that PD-1 blockade is effective and may have definite advantages in patients with resectable NSCLC. Therefore, the present study was conducted to evaluate the anti-tumor activity and safety of neoadjuvant PD-1 blocking mAbs used in combination with standard chemotherapy for potentially resectable (clinical stage IIA-IIIB) NSCLC patients for only Asian performed setting in two different oncology centers in China (Xi'an and Chongqing). We present the following article in accordance with the STROBE reporting checklist (available at http://dx.doi.org/10.21037/tlcr-21-130).

\section{Methods}

This study is a multinstitutional single-arm observational study, including 23 patients who received the neoadjuvant treatment in two different Chinese center study. Clinical stages IIA-IIIB NSCLC patients over the age of 18 years with were eligible for inclusion. All of the enrolled patients were scheduled to receive surgery within 4-6 weeks after neoadjuvant therapy that consisted in 3-4 cycles of a conventional chemotherapy regimen with PD-1 inhibitors on day 1 of each cycle according to the international consensus. The specific chemoimmunotherapy regimens are detailed in the Table 1. Additional inclusion criteria included eligibility for surgery, as indicated by auxiliary examination including lung function and blood gas analysis; no distant metastasis; and no contraindications for PD-1 inhibitor therapy. The patients' clinical staging was reviewed by 3 senior physicians and was confirmed if at least 2 of them agreed. Positron emission tomography/computed tomography (PET/CT) and immunohistochemicalPD-L1 detection were not necessary for inclusion. Patients with adenocarcinoma who had epidermal growth factor receptor $(E G F R)$ mutation or anaplastic lymphoma kinase $(A L K)$ rearrangement were excluded. The study was conducted in accordance with the Declaration of Helsinki (as revised in 2013). The study was approved by the Ethics Committee of Tangdu Hospital of the Fourth Military Medical University (202012-12-KY-02-XW-01) and informed consent was 
Table 1 Specific chemoimmunotherapy regimens received by each patient

\begin{tabular}{|c|c|c|c|c|c|c|}
\hline Patient no. & Chemoimmunotherapy & Cycles & Pathological type & $\begin{array}{l}\text { Response as per } \\
\text { RECIST v.1.1 }\end{array}$ & $\mathrm{pCR} / \mathrm{MPR}$ & $\begin{array}{l}\text { PD-L1 } \\
\text { (TPS) }\end{array}$ \\
\hline P1 & $\begin{array}{l}\text { Pemetrexed disodium }\left(500 \mathrm{mg} / \mathrm{m}^{2}, \mathrm{D} 1\right)+ \\
\text { cisplatin }\left(75 \mathrm{mg} / \mathrm{m}^{2}, \mathrm{D} 1\right)+\text { nivolumab }(360 \mathrm{mg}, \mathrm{D} 1)\end{array}$ & 3 & Adenocarcinoma & PR & $\mathrm{pCR}$ & $\mathrm{N}$ \\
\hline P2 & $\begin{array}{l}\text { Nab-paclitaxel }\left(260 \mathrm{mg} / \mathrm{m}^{2}, \mathrm{D} 1\right)+\text { nedaplatin } \\
\left(80 \mathrm{~m} / \mathrm{m}^{2}, \mathrm{D} 1\right)+\text { pembrolizumab }(200 \mathrm{mg}, \mathrm{D} 1)\end{array}$ & 3 & Squamous carcinoma & PR & & $5 \%$ \\
\hline P4 & $\begin{array}{l}\text { Nab-paclitaxel }\left(130 \mathrm{mg} / \mathrm{m}^{2}, \mathrm{D} 1,8\right)+\text { nedaplatin } \\
\left(80 \mathrm{~m} / \mathrm{m}^{2}, \mathrm{D} 1\right)+\text { nivolumab }(200 \mathrm{mg}, \mathrm{D} 1)\end{array}$ & 3 & Squamous carcinoma & PR & MPR & $\mathrm{N}$ \\
\hline P5 & $\begin{array}{l}\text { Nab-paclitaxel }\left(130 \mathrm{mg} / \mathrm{m}^{2}, \mathrm{D} 1,8\right)+\text { nedaplatin } \\
\left(80 \mathrm{~m} / \mathrm{m}^{2}, \mathrm{D} 1\right)+\text { nivolumab }(200 \mathrm{mg}, \mathrm{D} 1)\end{array}$ & 3 & Squamous carcinoma & PR & MPR & $\mathrm{N}$ \\
\hline P8 & $\begin{array}{l}\text { Gemcitabine }\left(1,000 \mathrm{mg} / \mathrm{m}^{2}, \mathrm{D} 1,8\right)+\text { nedaplatin } \\
\left(80 \mathrm{~m} / \mathrm{m}^{2}, \mathrm{D} 1\right)+\text { pembrolizumab }(200 \mathrm{mg}, \mathrm{D} 1)\end{array}$ & 2 & Squamous carcinoma & PR & MPR & $\mathrm{N}$ \\
\hline P9 & $\begin{array}{l}\text { Nab-paclitaxel }\left(130 \mathrm{mg} / \mathrm{m}^{2}, \mathrm{D} 1,8\right)+\text { nedaplatin } \\
\left(80 \mathrm{~m} / \mathrm{m}^{2}, \mathrm{D} 1\right)+\text { pembrolizumab }(200 \mathrm{mg}, \mathrm{D} 1)\end{array}$ & 4 & Squamous carcinoma & PR & $\mathrm{pCR}$ & $\mathrm{N}$ \\
\hline P10 & $\begin{array}{l}\text { Nab-paclitaxel }\left(130 \mathrm{mg} / \mathrm{m}^{2}, \mathrm{D} 1,8\right)+\text { nedaplatin } \\
\left(80 \mathrm{~m} / \mathrm{m}^{2}, \mathrm{D} 1\right)+\text { pembrolizumab }(200 \mathrm{mg}, \mathrm{D} 1)\end{array}$ & 3 & Squamous carcinoma & PR & & $5 \%$ \\
\hline P11 & $\begin{array}{l}\text { Nab-paclitaxel }\left(130 \mathrm{mg} / \mathrm{m}^{2}, \mathrm{D} 1,8\right)+\text { nedaplatin } \\
\left(80 \mathrm{~m} / \mathrm{m}^{2}, \mathrm{D} 1\right)+\text { pembrolizumab }(200 \mathrm{mg}, \mathrm{D} 1)\end{array}$ & 3 & Squamous carcinoma & PR & & $5 \%$ \\
\hline P15 & $\begin{array}{l}\text { Nab-paclitaxel }\left(130 \mathrm{mg} / \mathrm{m}^{2}, \mathrm{D} 1,8\right)+\text { nedaplatin } \\
\left(80 \mathrm{~m} / \mathrm{m}^{2}, \mathrm{D} 1\right)+\text { sintilimab }(200 \mathrm{mg}, \mathrm{D} 1)\end{array}$ & 4 & Squamous carcinoma & PR & & $1 \%$ \\
\hline P16 & $\begin{array}{l}\text { Nab-paclitaxel }\left(130 \mathrm{mg} / \mathrm{m}^{2}, \mathrm{D} 1,8\right)+\text { nedaplatin } \\
\left(80 \mathrm{~m} / \mathrm{m}^{2}, \mathrm{D} 1\right)+\text { sintilimab }(200 \mathrm{mg}, \mathrm{D} 1)\end{array}$ & 3 & Squamous carcinoma & SD & & $15 \%$ \\
\hline P17 & $\begin{array}{l}\text { Nab-paclitaxel }\left(130 \mathrm{mg} / \mathrm{m}^{2}, \mathrm{D} 1,8\right)+\text { nedaplatin } \\
\left(80 \mathrm{~m} / \mathrm{m}^{2}, \mathrm{D} 1\right)+\text { sintilimab }(200 \mathrm{mg}, \mathrm{D} 1)\end{array}$ & 2 & Squamous carcinoma & SD & & $<1 \%$ \\
\hline P18 & $\begin{array}{l}\text { Nab-paclitaxel }\left(130 \mathrm{mg} / \mathrm{m}^{2}, \mathrm{D} 1,8\right)+\text { nedaplatin } \\
\left(80 \mathrm{~m} / \mathrm{m}^{2}, \mathrm{D} 1\right)+\text { nivolumab }(200 \mathrm{mg}, \mathrm{D} 1)\end{array}$ & 4 & Adenocarcinoma & PR & $\mathrm{pCR}$ & $15.4 \%$ \\
\hline P19 & $\begin{array}{l}\text { Pemetrexed disodium (500 mg/m², D1) + cisplatin } \\
(75 \mathrm{mg} / \mathrm{m} 2, \mathrm{D} 1)+\text { sintilimab }(200 \mathrm{mg}, \mathrm{D} 1)\end{array}$ & 3 & Adenocarcinoma & SD & & $<1 \%$ \\
\hline P20 & $\begin{array}{l}\text { Nab-paclitaxel }\left(130 \mathrm{mg} / \mathrm{m}^{2}, \mathrm{D} 1,8\right)+\text { nedaplatin } \\
\left(80 \mathrm{~m} / \mathrm{m}^{2}, \mathrm{D} 1\right)+\text { pembrolizumab }(200 \mathrm{mg}, \mathrm{D} 1)\end{array}$ & 3 & Squamous carcinoma & PR & $\mathrm{pCR}$ & $\mathrm{N}$ \\
\hline
\end{tabular}

Table 1 (continued) 
Table 1 (continued)

\begin{tabular}{|c|c|c|c|c|c|c|}
\hline Patient no. & Chemoimmunotherapy & Cycles & Pathological type & $\begin{array}{l}\text { Response as per } \\
\text { RECIST v.1.1 }\end{array}$ & $\mathrm{pCR} / \mathrm{MPR}$ & $\begin{array}{l}\text { PD-L1 } \\
\text { (TPS) }\end{array}$ \\
\hline P21 & $\begin{array}{l}\text { Nab-paclitaxel }\left(130 \mathrm{mg} / \mathrm{m}^{2}, \mathrm{D} 1,8\right)+\text { nedaplatin } \\
\left(80 \mathrm{~m} / \mathrm{m}^{2}, \mathrm{D} 1\right)+\text { pembrolizumab }(200 \mathrm{mg}, \mathrm{D} 1)\end{array}$ & 3 & Squamous carcinoma & PR & & $\mathrm{N}$ \\
\hline P22 & $\begin{array}{l}\text { Paclitaxel }(175 \text { mg/m², D1) + carboplatin } \\
(A \cup C=5, D 1)+\text { nivolumab (360 mg, D1) }\end{array}$ & 2 & Squamous carcinoma & PR & & $\mathrm{N}$ \\
\hline
\end{tabular}

pCR, pathological complete response; MPR, major pathological response; PR, partial response; SD, stable disease; N, none detected; AUC, area under the curve.

taken from all individual participants.

\section{Study endpoints}

The primary endpoints of the study were the safety and efficacy of neoadjuvant chemotherapy combined with PD-1 inhibitors. The safety-related endpoints included adverse events (AEs) according to the Common Terminology Criteria for Adverse Events (CTCAE, v.4.0). The efficacyrelated endpoints included ORR according to the Response Evaluation Criteria in Solid Tumors (RECIST v.1.1), and postoperative pathological down-staging according to the eighth edition of the National Comprehensive Cancer Network (NCCN) guidelines for tumor-node-metastasis (TNM) staging of NSCLC.

The secondary study endpoints were pCR, which was defined as the complete absence of tumor cells, and MPR, which was defined as $<10 \%$ residual viable tumor (RVT).

\section{Statistical analysis}

Adverse events and feasibility were continuously monitored. Continuous variables are presented as means with standard deviation, while categorical variables are presented as frequencies.

\section{Results}

\section{Patient characteristics}

Twenty-three eligible candidates were recruited for this study. There were 22 men and one woman with just one of them with no smoking habits. A squamous histology and an adenocarcinoma were respectively, recorded in 19 and 4 of these patients with no driver mutations/translocation (EGFR or ALK etc.). Three cases were in IIA stage, 3 cases in IIB, 8 cases in IIIA, and 9 cases of IIIB stage (Table 2). Twenty patients underwent surgical resection Fourteen of them completed 3-4 cycles of chemo-immunotherapy as described elsewhere (method section) prior surgery; five further patients experienced grade 1 or 2 irAEs and where addressed to surgery after two treatment cycles while one last patient presented a lung abscess and underwent surgery after just one neo-adjuvant treatment cycle. All data on specific chemo-immunotherapy regimens are shown in Table 1. Three patients did not receive; in fact, two of them who completed the neoadjuvant program refused the risk of the surgery (Patients \#21 and \#23, Table 2) while another one (\#22) refused to complete the neoadjuvant program after he developed a severe (grade 3) hyperglycemia during the second chemotherapy cycle.

\section{Surgical treatment}

Among the 20 patients who underwent surgery, 11 patients underwent lobectomy, 5 patients underwent sleeve resection/bronchoplasty, 2 patients received bilobectomy, and 2 patients underwent pneumonectomy. The results for surgical treatment, morbidity, and mortality are reported in the Table 3 shows. Surgical methods included Da Vinci's/video-assisted thoracoscopic surgery (VATS) $(\mathrm{n}=14)$, conversion to thoracotomy $(\mathrm{n}=2)$, and thoracotomy $(n=4)$. Complete resection (R0) was achieved in $95 \%$ of patients $(19 / 20)$ who received surgery that in one case was considered as R1 resection due to the patient being lymphnode positive at the highest station. The median amount of blood lost was $212.5 \mathrm{~mL}(50-600 \mathrm{~mL})$, and the mean operative time was 250 minutes (65-390 minutes). 
Table 2 Baseline characteristics of the intention-to-treat population $(\mathrm{N}=23)$

\begin{tabular}{llll}
\hline Characteristics & Value & Surgery & Rejected surgery \\
\hline
\end{tabular}

Age, years

Median (range)

$\geq 60$ years

$<60$ years

Sex

Male

Female

BMI, kg/m² (mean)

History of smoking

Current/ex-smoker

Never-smoker

22

1

19

1

KPS

90

100

$\begin{array}{ll}21 & 19 \\ 2 & 1\end{array}$

Pathological type

Squamous carcinoma

Adenocarcinoma

$\begin{array}{ll}19 & 16\end{array}$

Clinical T stage

T1
T2
T4

Clinical N stage

$\begin{array}{lccc}\text { No } & 4 & 4 & 0 \\ \text { N1 } & 9 & 8 & 1 \\ \text { N2 } & 10 & 8 & 2\end{array}$

Clinical stage

\begin{tabular}{llll} 
IIA & 3 & 2 & 1 \\
IIB & 3 & 3 & 0 \\
IIIA & 8 & 8 & 0 \\
IIIB & 9 & 7 & 2 \\
\hline
\end{tabular}

\section{Safety}

The post-operative complications were considered to be unrelated to neoadjuvant therapy and are summarized in the Table 4. There was no 30- or 90-day mortality, or any
Table 3 Surgical procedures

\begin{tabular}{lc}
\hline Characteristics & Results \\
\hline Extent of surgery & 11 \\
Lobectomy & 2 \\
Bilobectomy & 5 \\
Sleeve resection/bronchoplasty & 2 \\
Pneumonectomy & \\
Surgical method & 14 \\
VATS/da Vinci & 2 \\
Conversion to thoracotomy & 4 \\
Thoracotomy & $250(65-390)$ \\
Operation time (min) & $212.5(50-600)$ \\
Bleeding (mL) & $11.125(6-22)$ \\
Hospital stay (days) & $4.18(0-7)$ \\
Chest tube duration (days) & 19 \\
Resection margins & 0 \\
R0 & 19 \\
R1 & \\
R2 & \\
\hline
\end{tabular}

postoperative arrhythmia episode. The occurrence of AEs is shown in detail in the Table 5. The most common grade 1 or 2 neoadjuvant treatment-related AEs were fatigue (39.1\%), alopecia (39.1\%), vomiting (34.7\%), leucopenia (30.4\%), neutropenia $(30.4 \%)$, and anorexia $(30.4 \%)$. The treatmentrelated grade 3 AEs included anorexia, vomiting, fatigue, alopecia, arthralgia, bone pain, and hyperglycemia. Three patients experienced typical immune-related AEs including grade 1-2 hyperthyroidism, grade 3 hyperglycemia, and grade 1-2 rash.

\section{Antitumor activity}

The activity of the neoadjuvant combination was evaluated according to the RECIST v.1.1 criteria after three/four treatment cycles. In particular, 17 out of the 23 showed a $\mathrm{PR}$, while 6 further patients presented a SD (Figure 1A). The postoperative pathology results showed a MPR (50\%) in 10 patients and a pCR $(30 \%)$ in 6 patients (Figure $1 A)$. A total of 15 patients $(75 \%)$ resulted pathologically downstaged after surgery. On the overall, our analysis showed a median progression-free-survival (PFS) of 11.3 months 
Table 4 Postoperative complications

\begin{tabular}{lc}
\hline Characteristics & $\mathrm{N}(\%)$ \\
\hline Intraoperative blood transfusion & $2(10 \%)$ \\
Death within 30 and 90 days & 0 \\
Heart failure & $1(5 \%)$ \\
Postoperative arrhythmia & 0 \\
Postoperative hoarseness & $1(5 \%)$ \\
Urinary tract infection or urinary retention & $1(5 \%)$ \\
\hline
\end{tabular}

Table 5 Adverse events

\begin{tabular}{lcccc}
\hline Characteristics & Any grade & Grade 1-2 & Grade 3 & Grade 4 \\
\hline Anemia & 3 & 3 & \\
Leukopenia & 7 & 7 & \\
Neutropenia & 7 & 7 & \\
Anorexia & 8 & 7 & 1 \\
Vomiting & 9 & 8 & 1 \\
Diarrhea & 2 & 2 & \\
Constipation & 1 & 1 & \\
Fatigue & 10 & 9 & 1 \\
Alopecia & 10 & 9 & 1 \\
Hyperthyroidism & 1 & 1 & \\
Lung abscess & 1 & 1 & \\
Rash & 1 & 1 & 1 \\
Arthralgia and & 2 & 1 & \\
bone pain & & & 1 \\
Hyperglycemia & 1 & & \\
\hline
\end{tabular}

(range, 3.1-18.7 months) (Figure 1B).

\section{Discussion}

This is a clinical trial testing that a neoadjuvant PD-1 blockade and chemotherapy combination for potentially resectable (clinical stage IIA-IIIB) NSCLC patients. Our study yielded promising results resulting in a MPR and a pCR of $50 \%$ and $30 \%$, respectively. This finding is of critical interest considering that previous studies in neoadjuvant setting, clearly show that both pCR and MPR are associated with survival benefits (8). For instance, Melek et al. examined a recorded a large database of
1,076 NSCLC patients who received surgery reporting a prolonged survival in those patients who achieved a pCR after neoadjuvant or induction therapy showing an outcome which was roughly the same as that recorded in patients diagnosed in a stage IB (1). These findings indicated a clinically significant association of pCR with 5-year OS (9). However, neither neoadjuvant chemotherapy, radiotherapy, nor TKI monotherapy has shown optimal pCR. The L'Intergroupe Francophone de Cancérologie Thoracique (IFCT) clinical trial reported that while only 41 (8.3\%) of the 492 study patients achieved pCR, the 5 -year OS of these patients reached $80.0 \%$, compared to $55.8 \%$ for patients without pCR (10). These clinical results clearly suggest that the OS outcomes of neoadjuvant patients are independently associated with pCR.

With the growing expansion of PD-1 immune checkpoint inhibitors in clinical practice, the CheckMate159 trial was conducted to investigate the effects of neoadjuvant therapy with the PD-1 inhibitor, nivolumab yielding an MPR rate of $45 \%$ and a pCR rate of $15 \%(n=3)(7)$. This trial also reported that the rates of $\mathrm{pCR}$ and MPR in patients treated with neoadjuvant nivolumab were much higher than those recorded in patients who received traditional neoadjuvant regimens with chemotherapy, TKI- or chemoradiation (11-13). Notably, in study concerning locally resectable NSCLC patients treated with neoadjuvant atezolizumab and chemotherapy, $10(33 \%)$ of 30 patients achieved a pCR which is in line with the results of our study (14).

Our data on AEs and complications indicate that the regimens used in this study were well tolerated, with no grade 4-5 AEs observed. AEs and complications occurred in this study at a similar frequency to those reported in the published data on neoadjuvant chemotherapy (15). In this study, we found that immune-related AEs could be well monitored and managed (16), which is similar to the observations made in the NADIM study (17). Only in 1 case did neoadjuvant therapy need to be halted after 1 cycle of treatment, with this patient's complication being lung abscess caused by obstructive pneumonia, which was unrelated to PD-1 inhibitor treatment. After stopping the neoadjuvant treatment, the patient underwent surgery. Interestingly, he achieved MPR, despite only receiving 1 cycle of chemoimmunotherapy. Therefore, this study fully demonstrated that combination neoadjuvant chemoimmunotherapy has good safety and efficacy for the treatment of stage IIA-IIIB NSCLC.

In the present study, the rates of conversion to thoracotomy, completed thoracotomy, and completed 
A

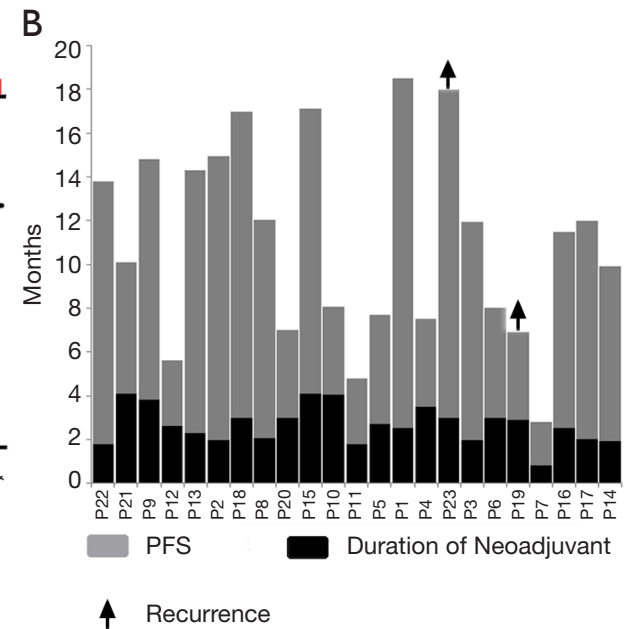

Figure 1 Waterfall plot. The horizontal dashed line represents partial response according to the RECIST v.1.1 criteria, and the different colors represent pathologic regression (A). Follow-up: duration of neoadjuvant therapy and PFS (B). pCR, pathological complete response; MPR, major pathological response; PFS, progression-free-survival.

pneumonectomy were $10 \%, 20 \%$, and $10 \%$ respectively, which are similar to rates reported in a neoadjuvant chemotherapy study (conversion to thoracotomy, 26.5\%; pneumonectomy, $17.6 \%$ ) (18). This finding suggests that neoadjuvant chemoimmunotherapy does not increase the complexity of the surgical procedure compared with neoadjuvant chemotherapy.

There are several limitations to this research. First, this study mainly focused on the safety and efficacy of neoadjuvant therapy due to the small number of patients. In the future, we hope to observe the 5-year OS after neoadjuvant chemoimmunotherapy in a larger patient cohort. Secondly, PD-L1 was detected in some but not all of the patients, while the tumor mutational burden was not recorded for any patient in this study. Furthermore, we did not attempt to reach a conclusion on the relationship between potential biomarkers of PD-L1 and neoadjuvant therapy, and this will be explored in future study. Thirdly, 7 of the 23 patients included refused to continue the neoadjuvant treatment after 1-2 cycles, which might have caused bias regarding the safety and efficacy data. However, the safety and efficacy data of patients who accepted surgery treatment were analyzed according to the different centers, drug treatments, and cycles (Appendix 1). There was no statistical difference for pCR of patients according to the different drugs and cycles.

\section{Conclusions}

The existing data suggest that, in the neoadjuvant setting, PD-1 inhibitors combined with chemotherapy can significantly improve the rates of pCR and MPR among patients with resectable NSCLC. The safety profile and surgical complications of these combination regimens are the same as those previously observed among patients who have received chemotherapy alone as a neoadjuvant therapy.

\section{Acknowledgments}

The authors appreciate the academic support from the AME Lung Cancer Collaborative Group.

Funding: This study was funded by the Tang Du Hospital Top-Level Talents Sponsorship Program, the Shaanxi Special Support Plan-Program for Leading Talents of Science and Technology Innovation, and the Tang Du Hospital's Special Fund for Medical Technology.

\section{Footnote}

Reporting Checklist: The authors have completed the STROBE reporting checklist. Available at http://dx.doi. org/10.21037/tlcr-21-130 
Data Sharing Statement: Available at http://dx.doi. org/10.21037/tlcr-21-130

Conflicts of Interest: All authors have completed the ICMJE uniform disclosure form (available at http://dx.doi. org/10.21037/tlcr-21-130). XY serves as an unpaid editorial board member of TLCR from Jun 2019 to Jun 2021. The other authors have no conflicts of interest to declare.

Ethical Statement: The authors are accountable for all aspects of the work in ensuring that questions related to the accuracy or integrity of any part of the work are appropriately investigated and resolved. The study was conducted in accordance with the Declaration of Helsinki (as revised in 2013). The study was approved by the Ethics Committee of Tangdu Hospital of the Fourth Military Medical University (202012-12-KY-02-XW-01) and informed consent was taken from all individual participants.

Open Access Statement: This is an Open Access article distributed in accordance with the Creative Commons Attribution-NonCommercial-NoDerivs 4.0 International License (CC BY-NC-ND 4.0), which permits the noncommercial replication and distribution of the article with the strict proviso that no changes or edits are made and the original work is properly cited (including links to both the formal publication through the relevant DOI and the license). See: https://creativecommons.org/licenses/by-nc-nd/4.0/.

\section{References}

1. Melek H, Çetinkaya G, Özer E, et al. Pathological complete response after neoadjuvant/induction treatment: where is its place in the lung cancer staging system? †. Eur J Cardiothorac Surg 2019;56:604-11.

2. Group NM-aC. Preoperative chemotherapy for non-smallcell lung cancer: a systematic review and meta-analysis of individual participant data. Lancet 2014;383:1561-71.

3. Topalian SL, Taube JM, Pardoll DM. Neoadjuvant checkpoint blockade for cancer immunotherapy. Science 2020;367:eaax0182.

4. Garassino MC, Gadgeel S, Esteban E, et al. Patientreported outcomes following pembrolizumab or placebo plus pemetrexed and platinum in patients with previously untreated, metastatic, non-squamous non-small-cell lung cancer (KEYNOTE-189): a multicentre, double-blind, randomised, placebo-controlled, phase 3 trial. Lancet Oncol2020;21:387-97.
5. Viteri S, Cabrera-Galvez C, Rosell R. Keynote 407: the combination of pembrolizumab and chemotherapy cracks the shell of squamous cell lung cancer. Transl Lung Cancer Res 2020;9:828-32.

6. Zhou Y, Lin Z, Zhang X, et al. First-line treatment for patients with advanced non-small cell lung carcinoma and high PD-L1 expression: pembrolizumab or pembrolizumab plus chemotherapy. J Immunother Cancer 2019;7:120.

7. Lommatzsch M, Bratke K, Stoll P. NeoadjuvantPD-1 Blockade in Resectable Lung Cancer. N Engl J Med 2018;379:e14.

8. Haque W, Verma V, Butler EB, et al. Pathologic nodal clearance and complete response following neoadjuvant chemoradiation for clinical N2 non-small cell lung cancer: Predictors and long-term outcomes. Lung Cancer 2019;130:93-100.

9. Hellmann MD, Chaft JE, William WN, et al. Pathological response after neoadjuvant chemotherapy in resectable non-small-cell lung cancers: proposal for the use of major pathological response as a surrogate endpoint. Lancet Oncol2014;15:e42-e50.

10. Mouillet G, Monnet E, Milleron B, et al. Pathologic complete response to preoperative chemotherapy predicts cure in early-stage non-small-cell lung cancer: combined analysis of two IFCT randomized trials. J Thorac Oncol 2012;7:841-9.

11. Zhang $\mathrm{Y}, \mathrm{Fu} \mathrm{F}, \mathrm{Hu} \mathrm{H}$, et al. Gefitinib as neoadjuvant therapy for resectable stage II-IIIA non-small cell lung cancer: A phase II study. J Thorac Cardiovasc Surg 2021;161:434-42.e2.

12. Zhang C, Li SL, Nie Q, et al. Neoadjuvant Crizotinib in Resectable Locally Advanced Non-Small Cell Lung Cancer with ALK Rearrangement. J Thorac Oncol 2019;14:726-31.

13. Cheedella NK, Suzuki A, Xiao L, et al. Association between clinical complete response and pathological complete response after preoperative chemoradiation in patients with gastroesophageal cancer: analysis in a large cohort. Ann Oncol 2013;24:1262-6.

14. Shu CA, Gainor JF, Awad MM, et al. Neoadjuvant atezolizumab and chemotherapy in patients with resectable non-small-cell lung cancer: an open-label, multicentre, single-arm, phase 2 trial. Lancet Oncol 2020;21:786-95.

15. Detterbeck FC, Socinski MA, Gralla RJ, et al. Neoadjuvant chemotherapy with gemcitabine-containing regimens in patients with early-stage non-small cell lung cancer. J Thorac Oncol 2008;3:37-45. 
16. Gu Y, Zhang H, Liu Z, et al. Different patterns of treatment-related adverse events of programmed cell death- 1 and its ligand- 1 inhibitors in different cancer types: A meta-analysis and systemic review of clinical trials. Asia Pac J Clin Oncol 2020;16:e160-78.

17. Provencio M, Nadal E, Insa A, et al. Neoadjuvant chemotherapy and nivolumab in resectable non-small-cell lung cancer (NADIM): an open-label, multicentre, single-

Cite this article as: Duan H, Wang T, Luo Z, Tong L, Dong X, Zhang Y, Afzal MZ, Correale P, Liu H, Jiang T, Yan X. Neoadjuvant programmed cell death protein 1 inhibitors combined with chemotherapy in resectable non-small cell lung cancer: an open-label, multicenter, single-arm study. Transl Lung Cancer Res 2021;10(2):1020-1028. doi: 10.21037/tlcr-21130 arm, phase 2 trial. Lancet Oncol 2020;21:1413-22.

18. Dy GK, Bogner PN, Tan W, et al. Phase II study of perioperative chemotherapy with cisplatin and pemetrexed in non-small-cell lung cancer. J Thorac Oncol 2014;9:222-30.

(English Language Editors: J. Grayand, J. Reynolds) 
Subgroup analysis: numerical analysis of different centers (Only in surgical patients)

\begin{tabular}{lcc}
\hline Characteristics & Xi'an $(\mathrm{n}=13)$ & Chongqing $(\mathrm{n}=7)$ \\
\hline RECIST & $10(76.9 \%)$ & $4(57.1 \%)$ \\
ORR & & \\
Postoperative pathology results & & \\
MPR & $8(61.5 \%)$ & $2(28.6 \%)$ \\
pCR & $4(30.7 \%)$ & $2(28.6 \%)$ \\
Extent of Surgery & & \\
Lobectomy & $7(53.8 \%)$ & $4(57.1 \%)$ \\
Bilobectomy & $1(7.7 \%)$ & $1(14.3 \%)$ \\
Sleeve resection/bronchoplasty & $4(30.7 \%)$ & $1(14.3 \%)$ \\
Pneumonectomy & $1(7.7 \%)$ & $1(14.3 \%)$ \\
Surgical method & & \\
VATS/da Vinci & $7(53.8 \%)$ & $7(100 \%)$ \\
Conversion to thoracotomy & $2(15.4 \%)$ & 0 \\
Thoracotomy & $4(30.7 \%)$ & 0 \\
\hline
\end{tabular}

ORR, overall response rate; $\mathrm{pCR}$, pathological complete response; MPR, major pathological response.

Subgroup analysis: numerical analysis of different drug treatments (Only in surgical patients)

\begin{tabular}{|c|c|c|c|}
\hline Characteristics & Sintilimab $(n=9)$ & Nivolumab $(n=4)$ & Pembrolizumab $(\mathrm{n}=7)$ \\
\hline \multicolumn{4}{|l|}{ RECIST } \\
\hline ORR & $3(33.3 \%)$ & $4(100 \%)$ & $7(100 \%)$ \\
\hline \multicolumn{4}{|l|}{ Postoperative pathology results } \\
\hline MPR & $2(22.2 \%)$ & $4(100 \%)$ & $4(57.1 \%)$ \\
\hline $\mathrm{pCR}$ & $1(11.1 \%)$ & $2(50 \%)$ & $3(42.8 \%)$ \\
\hline \multicolumn{4}{|l|}{$\mathrm{AE}$} \\
\hline Hyperthyroidism & $1(11.1 \%)$ & & \\
\hline Rash & & & $1(14.3 \%)$ \\
\hline \multicolumn{4}{|l|}{ Extent of surgery } \\
\hline Lobectomy & $5(55.6 \%)$ & $2(50 \%)$ & $4(57.1 \%)$ \\
\hline Bilobectomy & $1(11.1 \%)$ & & $1(14.3 \%)$ \\
\hline Sleeve resection/bronchoplasty & $2(22.2 \%)$ & $2(50 \%)$ & $1(14.3 \%)$ \\
\hline Pneumonectomy & $1(11.1 \%)$ & & $1(14.3 \%)$ \\
\hline \multicolumn{4}{|l|}{ Surgical method } \\
\hline VATS/da Vinci & $6(66.7 \%)$ & $4(100 \%)$ & $4(57.1 \%)$ \\
\hline Conversion to thoracotomy & $1(11.1 \%)$ & & $1(14.3 \%)$ \\
\hline Thoracotomy & 2 (22.2\%) & & 2 (28.6\%) \\
\hline
\end{tabular}

ORR, overall response rate; pCR, pathological complete response; MPR, major pathological response; AE, adverse event.

Subgroup analysis: numerical analysis of different cycles (Only in surgical patients)

\begin{tabular}{lcc}
\hline Characteristics & $1-2(n=6)$ & $3-4(\mathrm{n}=14)$ \\
\hline RECIST & $3(50 \%)$ & $11(78.6 \%)$ \\
ORR & & $7(50 \%)$ \\
Postoperative pathology results & $3(50 \%)$ & $5(35.7 \%)$ \\
MPR & $1(16.7 \%)$ & \\
pCR & & $1(7.1 \%)$ \\
AE & & \\
Hyperthyroidism & $1(16.7 \%)$ & $8(57.1 \%)$ \\
Rash & & $2(14.3 \%)$ \\
Extent of surgery & $3(50 \%)$ & $2(14.3 \%)$ \\
Lobectomy & & $2(14.3 \%)$ \\
Bilobectomy & $3(50 \%)$ & \\
Sleeve resection/bronchoplasty & & $9(64.2 \%)$ \\
Pneumonectomy & & $2(14.3 \%)$ \\
Surgical method & $5(83.3 \%)$ & $3(21.4 \%)$ \\
VATS/da Vinci & & $1(16.7 \%)$ \\
Conversion to thoracotomy & & \\
Thoracotomy &
\end{tabular}

RECIST, Response Evaluation Criteria In Solid Tumors; MPR, major pathological response; pCR, pathological complete response. 